\title{
Determinación de la tasa respiratoria de Eugenia stipitata Mc Vaugh (arazá) y Myrcyaria dubia HBK (camu-camu)
}

\author{
[Determination of respiratory rate of Eugenia stipitata Mc Vaugh (arazá) y \\ Myrcyaria dubia HBK (camu-camu)]
}

\author{
Littman Gonzáles*, Beder Aylas, Kike Paima \& Alberto Bazán \\ Facultad de Industrias Alimentarias, Universidad Nacional de la Amazonía Peruana, Calle Nauta 5ta cdra, Iquitos, Perú. \\ *e-mail: litgonri@hotmail.com
}

\begin{abstract}
Resumen
En el presente trabajo se determinó diariamente la tasa respiratoria de Eugenia stipitata Mc Vaugh (arazá) y Myrcyaria dubia HBK (camu-camu) a temperaturas de 10,20 y $28^{\circ} \mathrm{C}$, utilizando un respirómetro construido en el laboratorio. Eugenia stipitata Mc Vaugh, mostró un comportamiento climatérico, debido a su elevada tasa respiratoria y la presencia de picos climatéricos que coincidieron con el inicio de la maduración sensorial. Las tasas respiratorias registradas en los picos climatéricos fueron de $91,87 \mathrm{mgCO} / \mathrm{kg} . \mathrm{h}$ a los 21 días de almacenamiento a $10^{\circ} \mathrm{C}, 116,82 \mathrm{mgCO} / \mathrm{kg}$.h a los 7 días de almacenamiento a $20^{\circ} \mathrm{C}$ y 125,74 $\mathrm{mgCO}_{2} / \mathrm{kg}$.h a los 5 días de almacenamiento a $28^{\circ} \mathrm{C}$, respectivamente. Fue establecido que Eugenia stipitata Mc Vaugh a bajas temperaturas presenta menores tasas de respiración, prolongando de esta manera su vida comercial a $10^{\circ} \mathrm{C}$ hasta 3 semanas acompañado de óptimas características organolépticas, mientras que a temperaturas más altas como 20 y $28^{\circ} \mathrm{C}$ la vida comercial del producto desciende a 7 y 5 días respectivamente. Del mismo modo fue estudiado Myrcyaria dubia HBK el cual mostró un comportamiento no climatérico con bajas tasas de producción de $\mathrm{CO}_{2}$ con tendencia decreciente en el tiempo y ausencia de picos climatéricos. Cuando fue sometido a una temperatura de $10^{\circ} \mathrm{C}$, la tasa de respiración alcanzó 12 $\mathrm{mgCO}_{2} / \mathrm{kg}$.h, a los 20 días. El almacenamiento a mayores temperaturas como 20 y $28^{\circ} \mathrm{C}$ produjo un incremento en la tasa respiratoria de más del $200 \%$ alcanzando valores de 47 y $51 \mathrm{mgCO} / \mathrm{kg} \cdot \mathrm{h}$ al $10^{\circ}$ y $7^{\circ}$ día respectivamente.
\end{abstract}

Palabras clave: Tasa respiratoria, poscosecha, frutas climatéricas, frutas no climatéricas, $\mathrm{CO}_{2}$

\begin{abstract}
In the present work we determined daily respiratory rate of Eugenia stipitata Mc Vaugh (Arazá) and Myrcyaria dubia HBK (Camu-camu) at temperatures of 10,20 and $28^{\circ} \mathrm{C}$, using a respirometer constructed in the laboratory. Eugenia stipitata Mc Vaugh, showed a climacteric behavior, given high respiratory rate and the presence of climacteric peaks that coincided with the onset of sensory maturation. The respiratory rates registered in climacteric peaks were $91.87 \mathrm{mgCO} / \mathrm{kg}$.ha at 21 days of storage at $10^{\circ} \mathrm{C}, 116.82$ $\mathrm{mgCO} / \mathrm{kg}$.ha at 7 days of storage at $20^{\circ} \mathrm{C}$ and $125.74 \mathrm{mgCO} / \mathrm{kg}$.ha at 5 days of storage at $28^{\circ} \mathrm{C}$, respectively. It was established that Eugenia stipitata Mc Vaugh has lower respiration rates at low temperatures, thereby prolonging the shelf life at $10^{\circ} \mathrm{C}$ up to 3 weeks along with optimal organoleptic characteristics, while at higher temperatures such as 20 to $28^{\circ} \mathrm{C}$ commercial life descends to 7 and 5 days, respectively. Likewise, we studied Myrcyaria dubia HBK, which showed a climacteric behavior with low rates of $\mathrm{CO}_{2}$ production with decreasing trend over time and without climateric peaks. When subjected to $10^{\circ} \mathrm{C}$ respiration rates $12 \mathrm{mgCO} / \mathrm{kg}$.h were at 20 days. Storage at high temperatures such as 20 and $28^{\circ} \mathrm{C}$ caused an increase in respiratory rate of over $200 \%$ reaching values of 47 and $51 \mathrm{mgCO} / \mathrm{kg} . \mathrm{h}$ on the $10^{\text {th }}$ and $7^{\text {th }}$ day respectively.
\end{abstract}

Keywords: Respiratory rate, postharvest, climacteric fruits, non-climacteric fruits, $\mathrm{CO}_{2}$ 


\section{INTRODUCCIÓN}

Las frutas y vegetales en general mantienen un metabolismo activo aún después de la cosecha. La fotosíntesis prácticamente se detiene pero, sin embargo, los procesos de respiración prosiguen activos (Barreiro y Sandoval, 2002). Durante estos procesos, la producción de energía proviene de la oxidación de las propias reservas de almidón, azúcares y otros metabolitos. Una vez cosechado, el producto no puede reemplazar estas reservas que se pierden y la velocidad con que disminuyen es un factor de importancia en la duración de la vida poscosecha, por lo que la caracterización de la respiración es requerida para el diseño de almacenamientos idóneos que extiendan la vida útil del producto (Wills et al., 1998; Kader, 1992; Neves-Filho, 1991).
Se planteó como objetivo estudiar la actividad respiratoria en el tiempo a diversas temperaturas en arazá y camu-camu, mediante el empleo de un respirómetro construido en el Centro de Investigaciones de Recursos Naturales de la Amazonía (CIRNA), basado en el principio de recolección de $\mathrm{CO}_{2}$ sobre una solución analizable por titulación.

\section{MATERIALES Y MÉTODOS Diseño del respirómetro}

El respirómetro se construyó en el laboratorio de control de calidad del Centro de Investigaciones de Recursos Naturales (CIRNA), perteneciente a la Universidad Nacional de la Amazonía Peruana (UNAP). El prototipo (Figura 1) fue diseñado en base a lo reportado por Montes (2001) y Angueira et al., (2003).

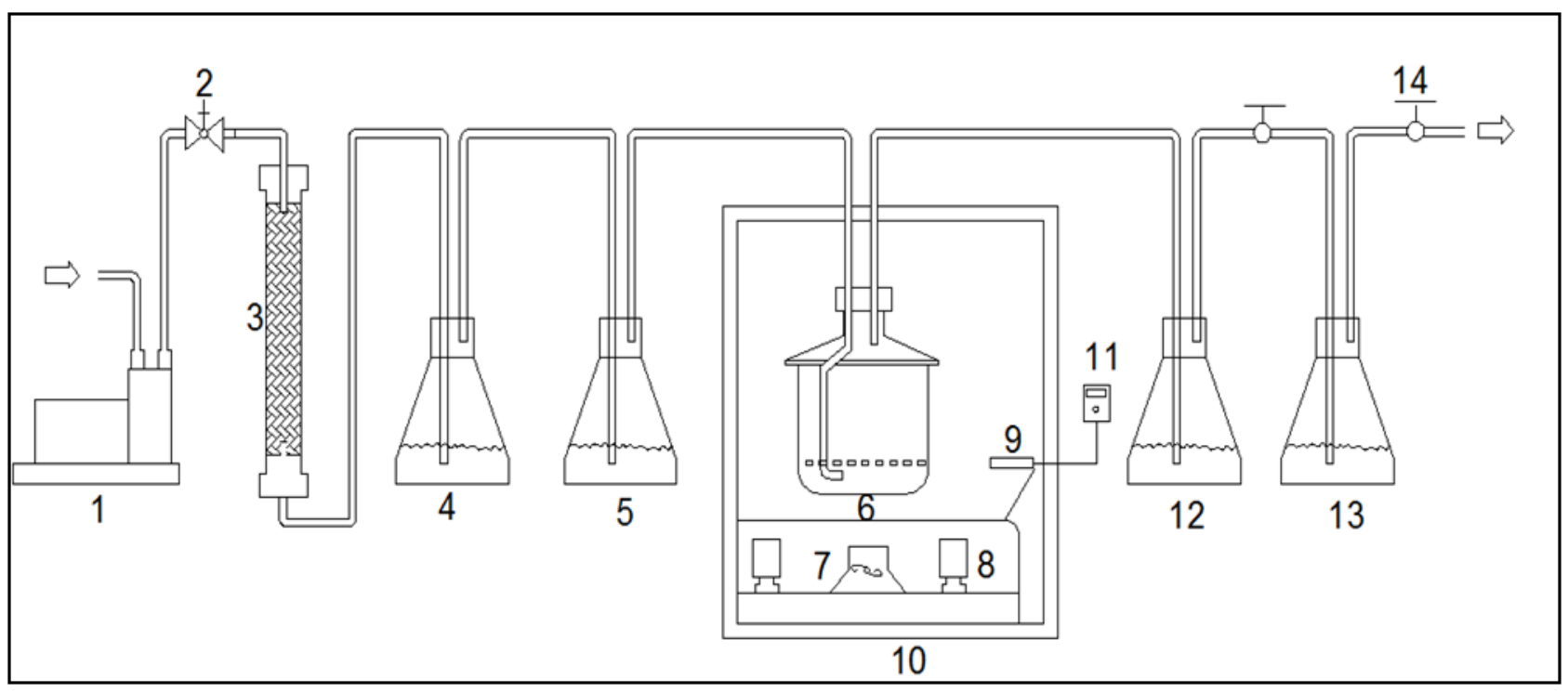

Figura 1. Diagrama del respirómetro empleado en la determinación de la tasa respiratoria de frutas y hortalizas.

1. Bomba de aire que suministra el flujo de aire por todo el sistema, a través de mangueras, para la remoción de los gases metabólicos producidos en la respiración y para proporcionar el $\mathrm{O}_{2}$ necesario para ésta, 2. Regulador de presión para control de flujo de aire suministrado, 3. Tubo de vidrio rellena de gel de sílice azul granulado (1-3mm) que elimina el vapor de agua contenido en el aire, evitando su reacción con el $\mathrm{NaOH}$, 4. Matraz con $80 \mathrm{ml}$ de $\mathrm{NaOH} 1 \mathrm{~N}$ para la absorción de $\mathrm{CO}_{2}$ contenido naturalmente en el aire, 5. Matraz con $80 \mathrm{ml}$ de agua destilada libre de $\mathrm{CO}_{2}$ que hidrata por burbujeo el aire libre de $\mathrm{CO}_{2}$ proveniente del matraz anterior, reponiendo así la humedad relativa eliminada por el gel sílice y reduciendo las pérdidas de peso por desecación de la fruta, 6. Desecador que simula el lugar de almacenamiento del vegetal, donde se coloca la fruta al cual se mide la tasa de respiración, 7. Ventilador para uniformizar el aire dentro de la cámara, 8. Lámparas para calentamiento, 9. Bulbo del termostato, 10. Sistema de refrigeración regulable a diferentes temperaturas donde se coloca el desecador conteniendo la fruta, 11. Termostato, 12. Matraz con $80 \mathrm{ml}$ de $\mathrm{HCl} 1 \mathrm{~N}$ cuya función es retirar la humedad sin absorber $\mathrm{CO}_{2}$ presente, pues debido al medio ácido no dispone de hidroxilos $(\mathrm{OH}-)$ para reaccionar con el $\mathrm{CO}_{2}$, 13. Matraz con $80 \mathrm{ml} \mathrm{de} \mathrm{NaOH}$ $1 \mathrm{~N}$ donde es absorbido el $\mathrm{CO}_{2}$ desprendido en el proceso de respiración, 14. Placas de Hoffman para control de salida de aire. 


\section{Determinación de la tasa respiratoria}

El $\mathrm{CO}_{2}$ desprendido durante el proceso de respiración de las frutas fue absorbido por una solución fuertemente alcalina. La titulación y subsecuente cuantificación del $\mathrm{CO}_{2}$ fue realizada según Angueira y col., (2003). La tasa respiratoria del Arazá y Camu-camu fue determinado a temperaturas de 10,20 y $28^{\circ} \mathrm{C}$, con HR de $90 \%$.

\section{RESULTADOS Y DISCUSIÓN Determinación de la tasa respiratoria del Arazá}

Los frutos de arazá fueron cosechados cuando alcanzaron la madurez fisiológica, presentaron una coloración verde clara uniforme en toda la superficie de la fruta (Figura 2).

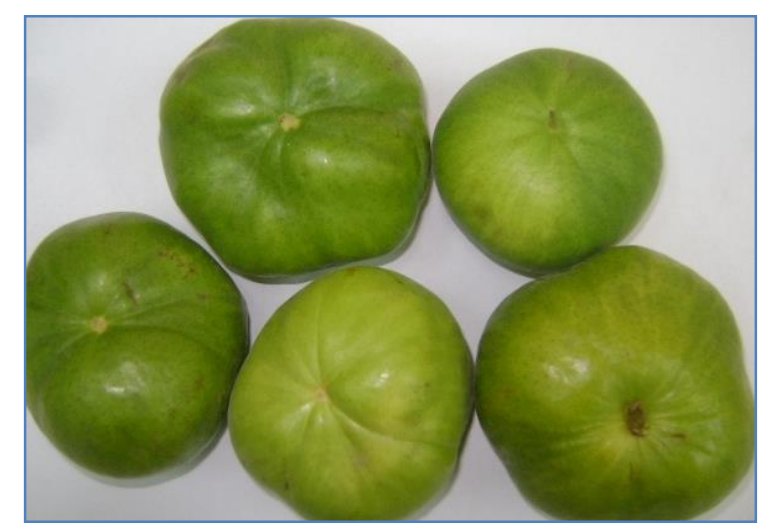

Figura 2. Frutos de Arazá al inicio del experimento

A $10^{\circ} \mathrm{C}$ el máximo climaterio se alcanzó a los 21 días (Figura 4). En el pico climatérico la tasa respiratoria fue de $91,87 \mathrm{mgCO}_{2} / \mathrm{Kg}$.h. La maduración fue incompleta e irregular, con presencia de manchas negras en la superficie de la fruta indicativo de haber sufrido daño por frío (Figura 3A). La FAO (1987) sostiene que la mayoría de las frutas tropicales experimentan daño por frío a temperatura entre 5 y $14^{\circ} \mathrm{C}$. Sin embargo, la parte interna del arazá se mantuvo en aceptables condiciones (Figura 3B).

A $20^{\circ} \mathrm{C}$ el pico climatérico se presentó a los siete días después de haber cosechado el fruto (Figura 4). Se observó una declinación en la evolución del $\mathrm{CO}_{2}$ inmediatamente después de la cosecha seguido por un crecimiento brusco.
Según Fernández (1990), el menor valor de generación de $\mathrm{CO}_{2}$ se designa como "mínimo pre-climatérico". El pico de respiración se designa como "máximo climatérico" y es seguido por un período de declinación en la actividad, designado como etapa "postclimatérico". En el pico de máximo climatérico la tasa respiratoria alcanzó valores de 116,82 $\mathrm{mg} . \mathrm{CO}_{2} / \mathrm{Kg}$.h. En estas condiciones, el arazá maduró uniformemente tanto externa como internamente (Figuras 3C y 3D) y no sufrió daño por frío ni ataques de microorganismos.

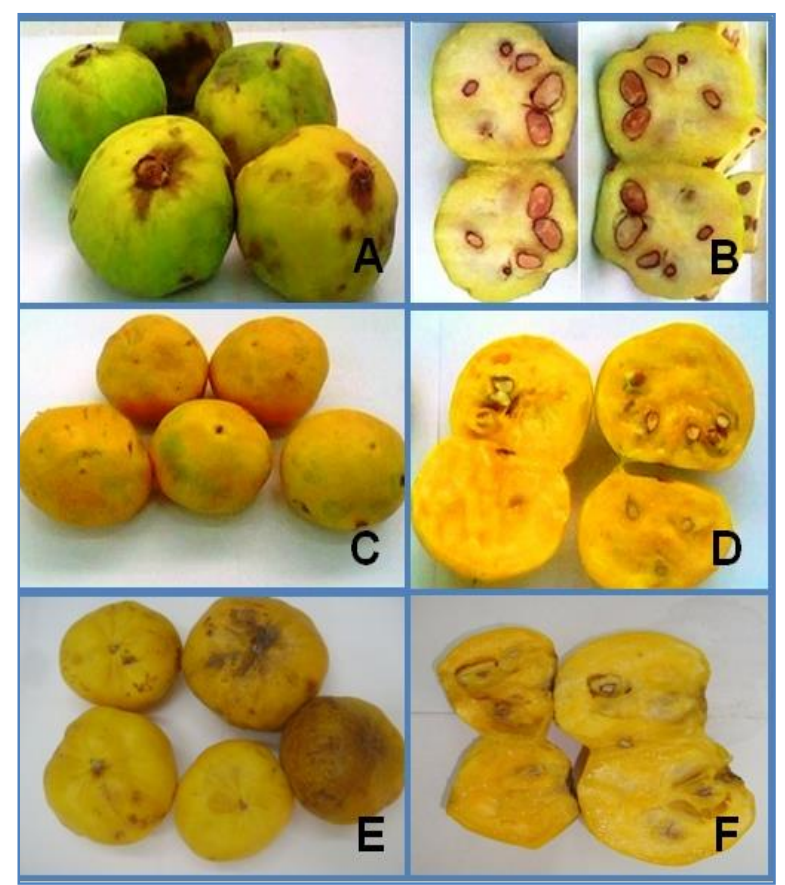

Figura 3. Vista exterior e interior de frutos de arazá almacenados a $10^{\circ} \mathrm{C}$ (A y B), $20^{\circ} \mathrm{C}$ (C y D), $28^{\circ} \mathrm{C}(\mathrm{E}$ y $\mathrm{F})$

A $28^{\circ} \mathrm{C}$ el pico climatérico se presentó al quinto día después de cosechado el fruto (Figura 4). La tasa respiratoria en su máximo climatérico fue de $125,74 \mathrm{mgCO} / \mathrm{Kg}$.h. En estas condiciones, la maduración inició a partir del tercer día y al quinto día alcanzó la madurez plena, produciéndose en seguida el deterioro propio de la senescencia (Figura 3E y 3F). La Figura 4 nos muestra que cuanto más bajo es la temperatura menor es la tasa de respiración, las formas de las curvas confirman que el arazá es una fruta climatérica. 


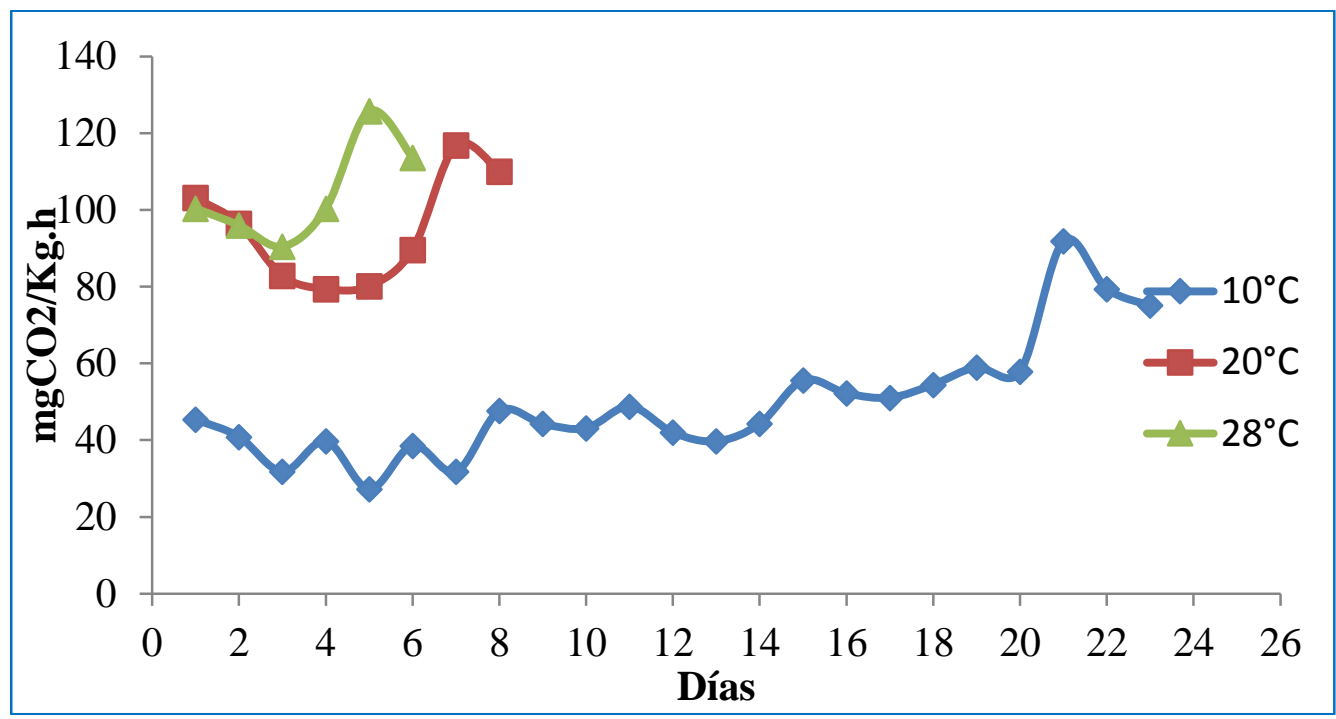

Figura 4. Tasa respiratoria del arazá "Eugenia stipitata"McVaugh durante su almacenamiento a 10, 20 y $28^{\circ} \mathrm{C}$.

\section{Determinación de tasa respiratoria del camu-camu}

Los frutos de camu-camu fueron cosechados en estado semi-maduro, presentaron una coloración verde clara combinado con coloración rojiza que abarcó alrededor del $50 \%$ de la superficie de la fruta (Figura 5 ). Se decidió trabajar con camu-camu semi-maduro debido a que en este estado la fruta contiene mayor contenido de vitamina $\mathrm{C}$ en comparación con el estado verde y maduro (Gonzales, 1987).

A $10^{\circ} \mathrm{C}$ la tasa respiratoria del camu-camu descendió en forma oscilante de 38 $\mathrm{mgCO}_{2} / \mathrm{Kg} . \mathrm{h}$ en el primer día a 12 $\mathrm{mgCO}_{2} / \mathrm{Kg}$.h al día 20 (Figura 7). El camucamu mantuvo su calidad externa (Figura 6A) hasta el día 15, a partir del día 16 aparecieron áreas hundidas y manchas de color marrón. No obstante, la fruta mantuvo la calidad interna hasta el día 20 del experimento (Figura 6B).

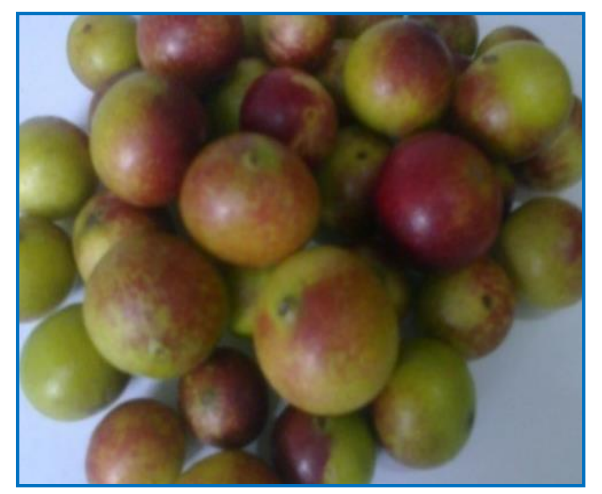

Figura 5. Frutos de camu-camu al inicio del experimento
A $20{ }^{\circ} \mathrm{C}$ la tasa respiratoria del camu-camu descendió de $58 \mathrm{mgCO}_{2} / \mathrm{Kg}$.h en el primer día a $47 \mathrm{mgCO}_{2} / \mathrm{Kg}$.h al décimo día (Figura 7), posteriormente se comenzó a notar manchas oscuras por toda la superficie de la cascara (Figura 6C y 6D).

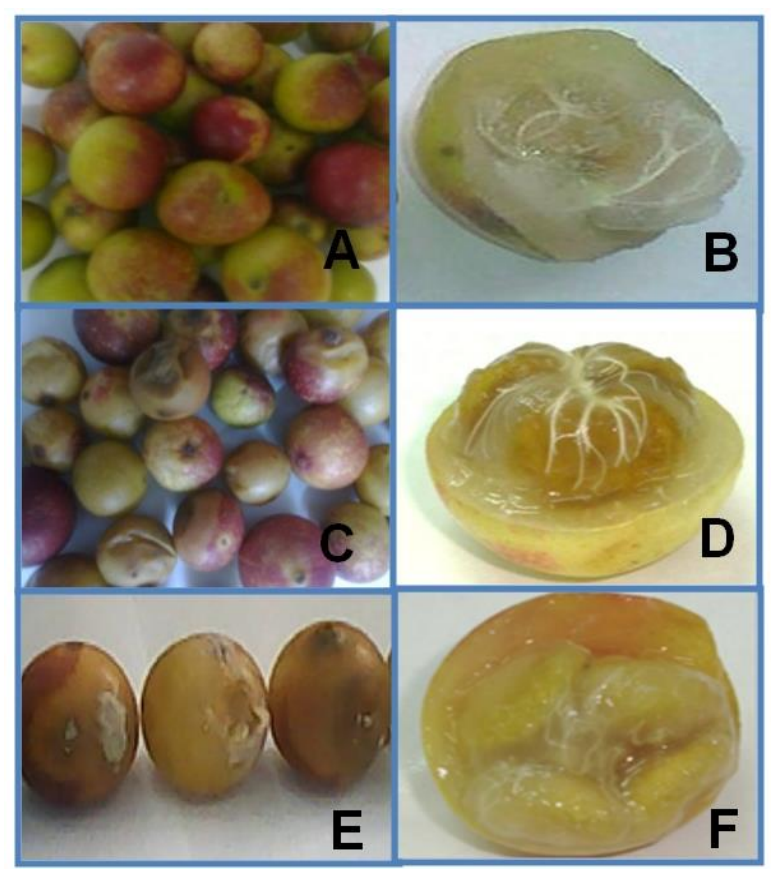

Figura 6. Vista exterior e interior de frutos camucamu almacenados a $10^{\circ} \mathrm{C}$ (A y B), $20^{\circ} \mathrm{C}$ (C y D), $28^{\circ} \mathrm{C}(\mathrm{E}$ y $\mathrm{F})$.

Cuando los frutos de camu-camu fueron almacenados a $28{ }^{\circ} \mathrm{C}$, la tasa respiratoria descendió de $62 \mathrm{mgCO}_{2} / \mathrm{Kg} . \mathrm{h}$, a 51 
$\mathrm{mgCO}_{2} / \mathrm{Kg}$.h al séptimo día (Figura 7). En estas condiciones, el deterioro se presentó a partir del cuarto día de almacenamiento, manifestándose con manchas negras y picaduras en la superficie de las frutas. Al sexto día aparecieron hongos alrededor de las picaduras (Figura 6E y 6F).

Es de resaltar que a 20 y $28^{\circ} \mathrm{C}$, la tasa de respiración del camu-camu fue $200 \%$ superior a lo obtenido a $10^{\circ} \mathrm{C}$. Este aumento de temperatura trajo consigo una reducción de la vida util de la fruta que concuerda con lo expresado por Seymour et al. (1993), que en muchos frutos no climatéricos por lo general existe una correlación entre tasas respiratorias elevadas y una corta vida útil pos-cosecha.

Los frutos de camu-camu analizados exhibieron una curva de respiración del tipo no climatérico sin evidencias de un pico o punto máximo definido guardando semejanza con los resultados obtenidos por Ortiz, O. J. y Suarez, R. A., (2006), quienes almacenaron camu-camu a $12{ }^{\circ} \mathrm{C}$, obteniendo tasas de respiración de 36 a $63 \mathrm{mgCO}_{2} / \mathrm{Kg} . \mathrm{h}$.

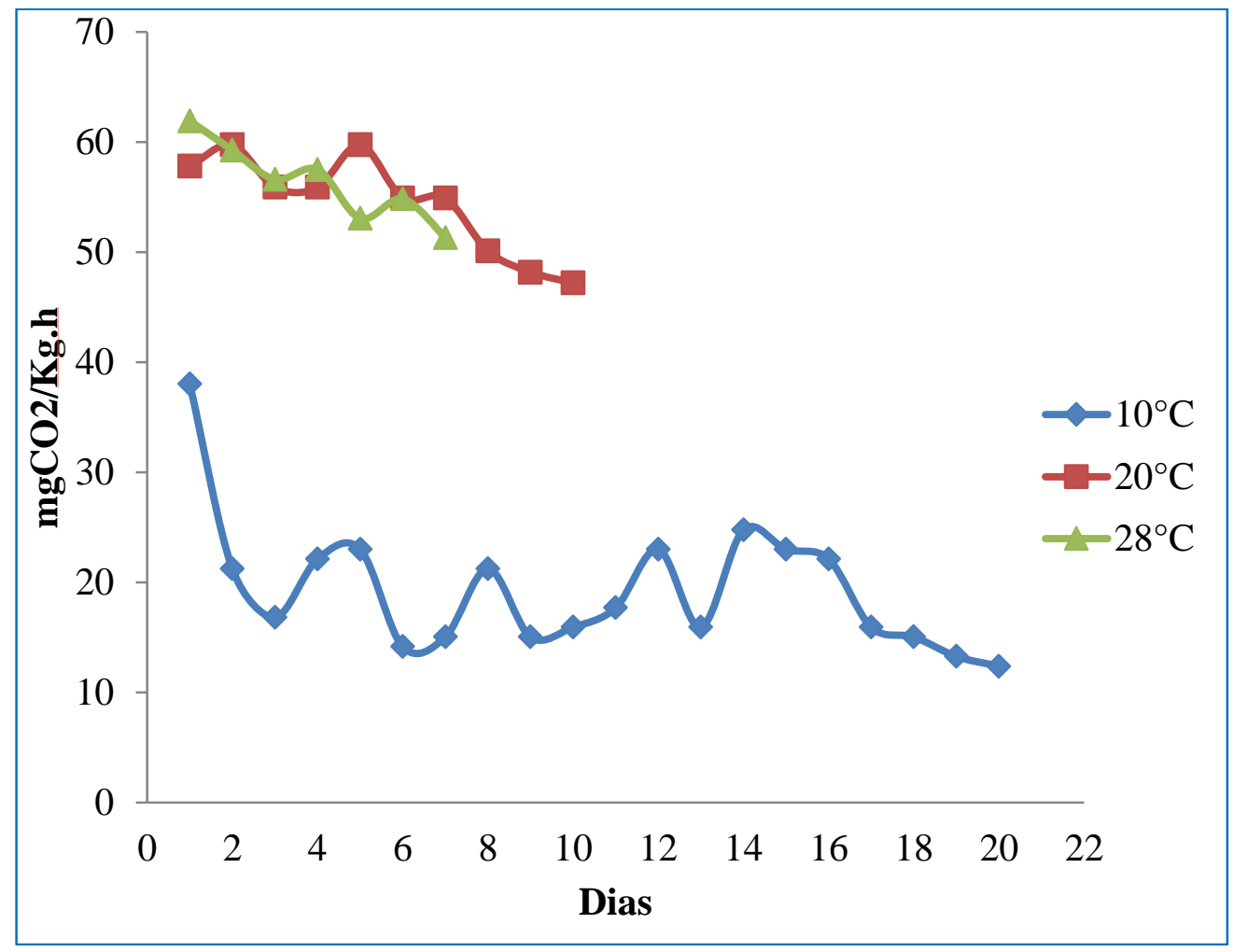

Figura 7. Tasa de respiración del Camu-camu a temperaturas de 10,20 y $28^{\circ} \mathrm{C}$.

\section{CONCLUSIONES}

El presente estudio confirma que el arazá es una fruta climatérica, muestra patrones de respiración elevados con tendencia ascendente y presencia de picos climatéricos, mientras que el camu-camu muestra un comportamiento no climatérico debido a sus bajas tasas de respiración con tendencia al descenso y ausencia de picos climatéricos. El comportamiento oscilante que presentan las curvas de respiración a $10^{\circ} \mathrm{C}$ tanto en arazá como en Camu-camu se debe probablemente a desórdenes fisiológicos ocasionados por el fenómeno conocido como daños por frío que se presentan en la mayoría de frutas tropicales.

\section{REFERENCIAS}

Angueira M, Sandoval A y Barreiro JA. 2003. Tasas de respiración en cuatro híbridos de pimentón (capsicum annum L.). INCI. 28: 593 - 596.

Barreiro JA, Sandoval AJ. 2002. Operaciones de conservación de alimentos por baja temperaturas. Editorial Equinoccio. Venezuela. 21 - $27 \mathrm{p}$.

FAO - Organización de las Naciones Unidas para la Agricultura y la Alimentacion. 1987. Manual para el mejoramiento del manejo poscosecha de frutas y hortalizas. 
Fernández y Bosco 1990. Pós-colheita de frutos e hortaliças. Fisiologia e Manuseio. Escuela superior de Agricultura de Lavras.

Gonzales L. 1987. Estudio Técnico Sobre la Elaboración de Conservas de Camu-camu "Myrciaria dubia" Tesis de Pre-Grado. Univ. Nacional de la Amazonia Peruana. Perú.

Kader AA. 1992. Postharvest technology of horticultural crops. Second Edition. University of California.

Montes J y Arévalo S. 2001. Determinación del calor de respiración de frutas por el método de titulación. RAIA. V.2.

Neves-Filho LC. 1991. Armazanamento de géneros e productos alimentícios. Governo do Estado de São Paulo.
Ortiz OJ, Suárez RA. 2006. Determinación de las condiciones óptimas de almacenamiento del fruto camu camu (myrciria dubia h.b.k.) en atmósferas Modificadas. Bogotá - Colombia.

Seymour GB, Taylor JE, Tucker GA. 1993. Biochemistry of fruit ripening. London, Chapman and Hall. 454 p.

Wills R, McGlasson D, Graham D y Joyce D. 1998. Introducción a la fisiología y manipulación poscosecha de frutas, hortalizas y plantas ornamentales. $2^{\text {da }}$. Edición. Editorial Acribia S.A. España. 30 44p. 